\title{
Machine learning identifies novel markers predicting functional decline in older adults
}

Kate E. Valerio ${ }^{1}$, Sarah Prieto ${ }^{1}$, Alexander N. Hasselbach ${ }^{1}$, Jena N. Moody ${ }^{1}$, Scott M. Hayes ${ }^{1,2}$, Jasmeet P. Hayes ${ }^{1,2}$, *the Alzheimer's Disease Neuroimaging Initiative

${ }^{1}$ Department of Psychology, The Ohio State University, Columbus, OH 43210, USA

${ }^{2}$ Chronic Brain Injury Initiative, The Ohio State University, Columbus, OH 43210, USA

* Data used in the preparation of this article were obtained in part from the Alzheimer's Disease Neuroimaging Initiative (ADNI) database (www.long.ucla.edu/ADNI). As such the investigators within the ADNI contributed to the design and implementation of ADNI and/or provided data but did not participate in analysis or writing of this report. ADNI investigators are listed at www.loni.ucla.edu/ADNI/Collaboration/ADNI-Authorship_list.pdf.

Correspondence to: Jasmeet P. Hayes

The Ohio State University

1835 Neil Avenue

Columbus, OH 43210, USA

E-mail: hayes.1075@osu.edu 


\section{Abstract}

The ability to carry out instrumental activities of daily living, such as paying bills, remembering appointments, and shopping alone decreases with age, yet there are remarkable individual differences in the rate of decline among older adults. Understanding variables associated with decline in instrumental activities of daily living is critical to providing appropriate intervention to prolong independence. Prior research suggests that cognitive measures, neuroimaging, and fluid-based biomarkers predict functional decline. However, a priori selection of variables can lead to the over-valuation of certain variables and exclusion of others that may be predictive. In the present study, we used machine learning techniques to select a wide range of baseline variables that best predicted functional decline in two years in individuals from the Alzheimer's Disease Neuroimaging Initiative dataset. The sample included 398 individuals characterized as cognitively normal or mild cognitive impairment. Support vector machine classification algorithms were used to identify the most predictive modality from five different data modality types (demographics, structural MRI, fluorodeoxyglucose-PET, neurocognitive, and genetic/fluid-based biomarkers). In addition, variable selection identified individual variables across all modalities that best predicted functional decline in a testing sample. Of the five modalities examined, neurocognitive measures demonstrated the best accuracy in predicting functional decline (accuracy $=74.2 \%$; area under the curve $=0.77$ ), followed by fluorodeoxyglucose-PET (accuracy $=70.8 \%$; area under the curve $=0.66$ ). The individual variables with the greatest discriminatory ability for predicting functional decline included partner report of language in the Everyday Cognition questionnaire, the ADAS13, and activity of the left angular gyrus using fluorodeoxyglucose-PET. These three variables collectively explained $32 \%$ of the total variance in functional decline. Taken together, the machine learning model identified novel biomarkers that may be involved in the processing, retrieval, and conceptual integration of semantic information and which predict functional decline two years 
after assessment. These findings may be used to explore the clinical utility of the Everyday Cognition as a non-invasive, cost and time effective tool to predict future functional decline.

Keywords: ADNI, angular gyrus, Everyday Cognition, IADL, machine learning

Abbreviations: A $\beta=$ amyloid beta; ADAS=Alzheimer's Disease Assessment Schedule; ADNI=Alzheimer's Disease Neuroimaging Initiative; ANRT=Adult National Reading Test; APOE=Apolipoprotein E; AUC=area under the curve; BDRS=Blessed Dementia Rating Scale; $\mathrm{BMI}=$ body mass index; $\mathrm{CDR}=\mathrm{Clinical}$ Dementia Rating; EcogSP=Everyday Cognition-Study Partner; FAQ=Functional Activities Questionnaire; FDG=fluorodeoxyglucose; fMRI=functional MRI; IADL=instrumental activities of daily living; MMSE=Mini-Mental Status Examination; MoCA=Montreal Cognitive Assessment; p-tau=phosphorylated tau; PHS=polygenic hazard score; RAVLT=Rey Auditory Verbal Learning Test; ROC=receiver operating characteristic; ROI=region of interest; SUV=standardized uptake value; $\mathrm{SVM}=$ support vector machine; VIF=variance inflation factor 


\section{Background}

The ability to carry out instrumental activities of daily living (IADLs), such as paying bills, remembering appointments, and shopping alone, decreases with age. An estimated $26 \%$ of community-dwelling older adults between 70 and 79 experience functional impairment in at least one domain (e.g., completing chores, managing money, preparing meals), increasing to $35 \%$ of adults between 80 and 89 (Seeman et al., 2010). Declines in IADLs are associated with Alzheimer's disease (McKhann et al., 2011), lower quality of life (Teng et al., 2012), depression (López et al., 2014), and diminished physical functioning (Vaughan et al., 2016). However, IADL ability varies widely among older adults; whereas some show reduced functional independence, others demonstrate preserved functional independence. As the population continues to age and life expectancy increases (Christensen et al., 2009), understanding variables associated with decline and preserved ability to carry out IADLs will be critical to provide appropriate and timely intervention.

A small set of studies have examined neurobiological and cognitive factors associated with longitudinal functional decline across the spectrum of normal and pathological aging. One study demonstrated that CSF markers of neurodegenerative pathology-i.e., tau, phosphorylated-tau (p-tau) and amyloid beta (A $\beta)$-predicted functional decline at 36 months in cognitively normal subjects and individuals with mild cognitive impairment (MCI; Okonkwo et al., 2010a), while another study found that whole brain atrophy based on structural MRI and Apolipoprotein E (APOE) $\varepsilon 4$ status predicted functional decline in MCI (Okonkwo et al., 2010b). Fluorodeoxyglucose (FDG)-PET and cognitive performance have also been shown to be associated with functional decline (Royall et al., 2004; Farias et al., 2009; Landau et al., 2011; Thomas et al., 2017). Taken together, the emerging literature has outlined a variety of neurobiological and cognitive factors that are associated with subsequent functional decline. However, some important limitations characterize previous work. First, variables included in 
prediction analyses were selected a priori in these studies, potentially leading to overvaluation of some variables and oversight of others that may explain greater variance in functional decline. Second, prior work has not included testing/validation samples or employed permutation testing, limiting generalizability of findings. Finally, studies examining genetic associations of functional decline have conducted candidate gene analyses, rather than considering polygenic effects, which have been shown to add predictive value to understanding many complex traits and diseases (Lewis and Vassos, 2017).

To address these limitations, we implemented machine learning techniques to identify variables at a baseline visit that were associated with functional decline within 24 months later. This data-driven approach is designed to give equal consideration to all available variables and allows the relationships between variables to determine the final model. Such approaches prevent biased experimenter expectancies from influencing results and are ideal for studying a facet as highly dimensional as human aging. Moreover, this technique can help identify risk and resilience factors from a large set of variables to allow a more targeted approach to treatment or prevention, providing a more individualized approach to understanding functional decline (Schumann et al., 2019). Although prior research has used machine learning to diagnose and predict conversion to Alzheimer's disease as well as Alzheimer's disease pathology (Cui et al., 2011; Gray et al., 2013; Apostolova et al., 2015; Ritter et al., 2015; Bucholc et al., 2019), this approach has yet to be implemented to examine functional decline. Functional decline is considerably more prevalent among older adults ( $26 \%$ of individuals between 70 and 79; Seeman et al., 2010) compared to Alzheimer's disease (9.7\% of individuals over the age of 70; Qiu et al., 2009), highlighting the need to examine variables predictive of functional decline.

In the current analyses, we implemented support vector machine (SVM) algorithms to construct models of functional decline at 24 months following baseline assessment using 
multiple data modalities (demographics, MRI, FDG-PET, neurocognitive, and genetic/fluidbased biomarkers) available in the Alzheimer's Disease Neuroimaging Initiative (ADNI) database. ADNI is a multicenter study that includes a sample of deeply characterized older adults. By grouping variables into modalities based on collection method, we sought to examine the extent to which less invasive methods of data collection (e.g., neurocognitive tests) have discriminative power equal to or better than more invasive markers of functional decline (e.g., CSF markers). In addition, we evaluated variables across modalities to identify the individual variables that were most associated with functional decline. Included in these variables was a polygenic risk score for Alzheimer's disease, which takes into account the effects of many genes. Finally, we take the selected variables and validate their effect in a separate sample to provide evidence of the generalizability of their predictive ability. The goals of this study were twofold: 1) Use machine learning techniques to identify data modalities and variables most predictive of functional decline in an unbiased and data-driven way; 2) Test those variables in an independent sample and examine their influence on future decline using multiple regression models.

\section{Materials and Methods}

\section{Subjects}

Data used for this manuscript were obtained from the ADNI database (adni.loni.usc.edu). ADNI is a large-scale longitudinal study that began in 2004 as a public-private partnership led by Principal Investigator Michael W. Weiner, MD. Participants were recruited to examine serial MRI, PET, biomarkers, clinical assessment, and neuropsychological assessment to measure the progression of MCI and Alzheimer's disease. Additional information can be found at adni.loni.usc.edu. Our analyses included 398 adults with preserved functional performance, as indicated by a diagnosis of normal cognition or MCI, and who completed the Functional Activity Questionnaire (FAQ) at a baseline and 24-month visit. Participants included in 
analyses were missing no more than $5 \%$ of all analyzed variables. Remaining missing data were imputed via simple random sampling ( $<1 \%$ of total data).

Study procedures were approved by site-specific Institutional Review Boards and all participants and/or authorized representatives provided written informed consent consistent with the Declaration of Helsinki. For more information about the diagnostic criteria used in ADNI, see the General Procedures Manual at adni.loni.usc.edu.

\section{Data types}

\section{Outcome variable}

The FAQ is a questionnaire that was designed to monitor functional changes in older adults and is considered the gold standard for assessing IADLs in an aging population (MalekAhmadi et al., 2015). It assesses ten items on a scale from 0-3, with higher scores indicating greater levels of impairment. Total scores range from 0 to 30 . It has demonstrated high reliability and sensitivity (85\%) in research and clinical settings (Pfeffer et al., 1982) and is useful for differentiating between cognitively normal and dementia patients (Pfeffer et al., 1982) as well as between MCI and Alzheimer's disease (Kaur et al., 2016), and MCI and normal cognition (Teng et al., 2012). In order to capture subtle changes in functioning, individuals who had a difference score greater than or equal to one point between their baseline and 24-month assessment were considered to have a decrease in functioning. This corresponds with any decrease in ability to carry out any of the ten items assessed, and previous research has shown that any dependence on others for any IADL is significantly associated with increased risk of developing Alzheimer's disease, as early as five years prior to diagnosis (Pérès et al., 2008). All other individuals were characterized as having either stable or improving functioning.

\section{Predictor variables}


A total of 508 variables were included in analyses, organized into five data modalities (demographics, MRI, FDG-PET, neurocognitive, genetic/fluid markers). These variables were also assessed for their individual contributions to functional decline. In total, there were 26 demographic variables, 341 MRI variables, 30 FDG-PET variables, 71 neurocognitive variables, and 40 genetic/fluid-based biomarker variables.

\section{Demographics}

Twenty-six demographic variables included age, sex, education, race, ethnicity, marital status, body mass index (BMI), baseline diagnosis ( $\mathrm{CN}$ or $\mathrm{MCI})$, and medical history. Medical history diagnoses were classified into one of 18 categories of illnesses- psychiatric, neurologic, ears/nose/throat (ENT), cardiac, respiratory, hepatic, dermatologic, musculoskeletal, endocrine/metabolic, gastrointestinal, blood, renal, allergic, alcohol-related, drug-related, smoking related, malignant, or surgical.

\section{MRI}

T1-weighted images were acquired on ADNI-approved 3T scanners and processed crosssectionally using the 2010 Desikan-Killiany atlas with FreeSurfer image analysis suite, version 5.1. For more information, see Supplementary Material. This included 69 cortical volume measurements (68 unilateral volumes from the left and right hemispheres and intracranial volume), 16 hippocampal subfield volume measurements, 50 subcortical volume measurements, 70 surface area measurements, and 136 thickness measurements. All volume measurements were corrected for intracranial volume (Buckner et al., 2004).

\section{Fluorodeoxyglucose-PET (FDG-PET)}

For more information on data collection and processing, see Supplementary Material. Metrics of regions of interest (ROIs) available through ADNI were previously determined from a metaanalysis that identified regions which significantly differed between MCI and Alzheimer's disease and predicted cognitive decline (Landau et al., 2011). Metrics of standardized uptake 
value (SUV) in five regions were included: the right temporal gyrus, left temporal gyrus, right angular gyrus, left angular gyrus, and posterior cingulate. Metrics of each region included the mean, median, mode, maximum, minimum, and standard deviation SUV of each ROI, for a total of 30 variables. For these variables, SUV refers to the ratio of the concentration of radioactive glucose analog in an ROI to the concentration in the rest of the body, corrected for the weight of the patient. It is a proxy for glucose metabolism in the brain, with larger SUVs suggesting increased glucose uptake and increased brain activity.

\section{Neurocognitive measures}

General cognition, executive function, learning and memory, language, and processing speed were assessed with 13 standardized neuropsychological tests. Seventy-one neurocognitive variables were derived from these 13 tests; this included subtests when available in the NEUROBAT.csv file from ADNI. Supplemental table 1 lists the specific tests used for each domain.

\section{Biological samples}

A single polygenic hazard score (PHS) for Alzheimer's disease was calculated using 31 single nucleotide polymorphisms and two APOE variants (Desikan et al., 2017). CSF was collected via lumbar puncture and analyzed for three variables: $A \beta_{1-42}$, tau, and $p$-tau. Blood plasma and urine serum were collected from participants and underwent standard clinical laboratory tests at the University of Pennsylvania Penn Biomarker Core (Trojanowski et al., 2010). Thirty-six variables from tests on blood plasma and urine serum were included in these analyses. For more information, see Supplementary Material.

\section{Analytic methods}

\section{Training and testing sample}

Analyses were run in R (3.6.1; RC Team, 2013) using the "caret" package (Kuhn, 2020). The sample was partitioned into two groups, a training sample (70\% of sample) and a testing sample 
Predicting functional decline

( $30 \%$ of sample), using the createDataPartition function. This function assigned participants to one of two groups, while ensuring that both groups had equal proportions of individuals who were characterized as declining functionally. Training and testing samples were compared using Mann-Whitney-U tests for continuous variables and Fisher's exact test for categorical variables. Nonparametric tests were used given that the properties required of parametric tests were not satisfied.

Fig. 1 shows the sequence of variable selection and variable evaluation. Briefly, the training sample was used to identify variables with the most predictive ability or highest variable importance as well as model tuning. The testing sample was used to evaluate model performance and generate receiver operating characteristic (ROC) curves, as well as the variance in functional decline explained by the selected predictor variables. 
Fig. 1. Flowchart illustrating process of variable selection and data analysis

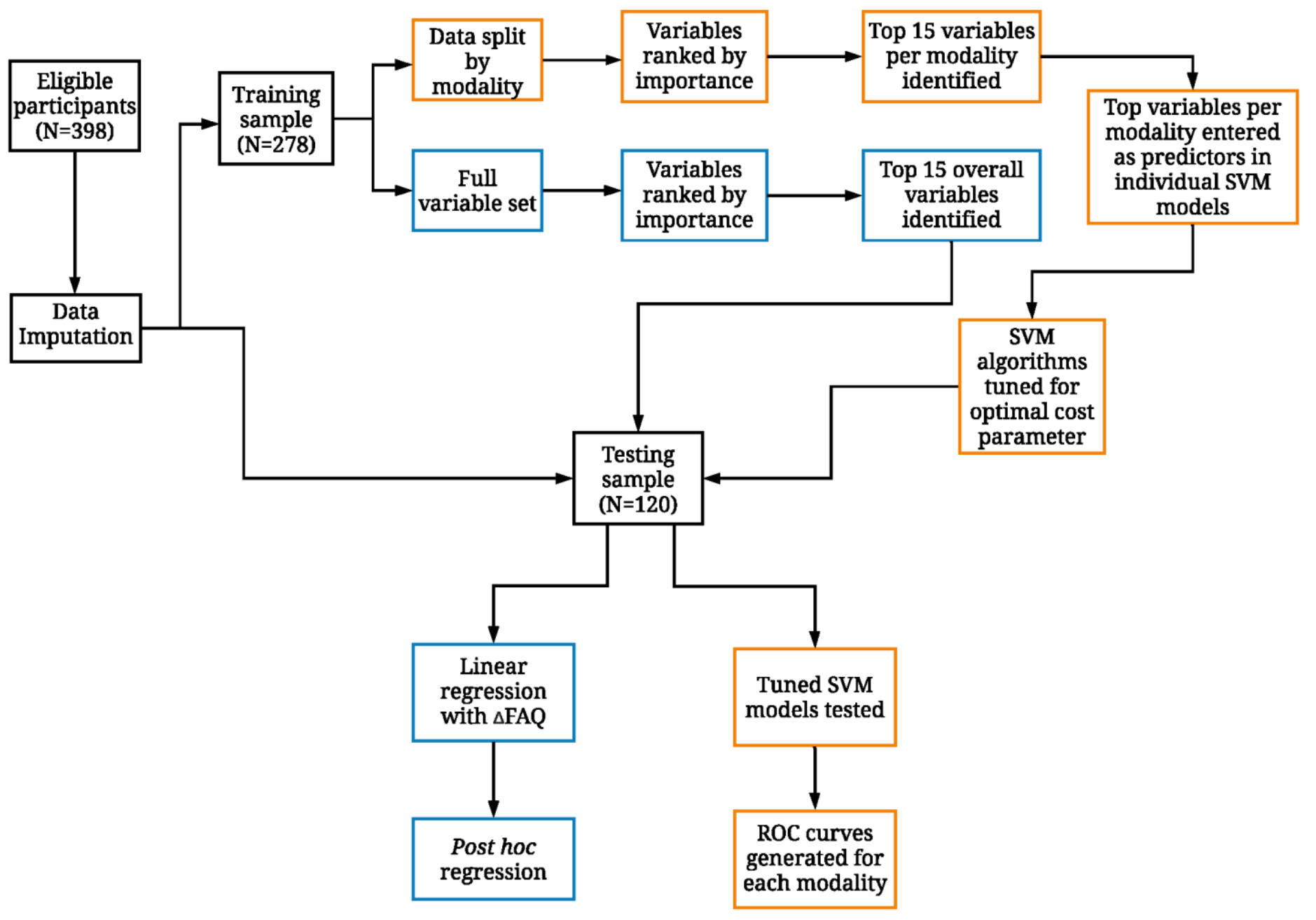

398 eligible participants were identified in the first step and missing data were imputed. In the second step, the data were split into the training sample and testing sample. Two parallel processes were run in the training sample. In the first process, shown in orange, data were split into each of the five modalities. Within each modality, the top 15 most predictive variables were identified by their individual variable importance. These selected variables were then entered as predictors into individual SVM models, one for each modality.

Within these individual SVM models, the models were trained for the optimal cost parameter. This final model was then tested using the testing sample. In the testing sample, ROC curves were generated for each modality. In the second process in the training sample, shown in blue, all variables from all modalities were included together. The top 15 most predictive variables were identified by the individual variable importance. These selected variables were then entered as predictors into a linear regression in the testing sample. Also in the testing sample, post hoc hierarchical regression analyses were conducted to better understand the relationship between significant predictors.

Abbreviations: FAQ=Functional Activities Questionnaire; ROC=receiver operating characteristics; $\mathrm{SVM}=$ support vector machine 


\section{Data modality analysis}

\section{Variable selection}

To determine which of the five data modalities (i.e., demographics, MRI, FDG-PET, neurocognitive, genetic/fluid markers) best predicted functional decline, the top 15 variables from each modality were first identified by ranking their importance to the model's prediction. Variable importance scores were calculated using the varImp function in the "caret" package. For an SVM algorithm, this function works by considering individually each variable in a model and defining a line that most accurately separates the two classes, using SVM; see below for more information about how SVM defines a line or hyperplane. This classification boundary is then systematically varied to adjust the ratio of true positives to false positives. The resulting ratios are plotted to generate an ROC curve representing the trade-off between sensitivity and specificity for the variable. The area under the curve (AUC) thus summarizes the variable's susceptibility to noise, with higher values suggesting greater discrimination ability. AUC was therefore used to determine variables with the greatest predictive ability. For more information about ROC curves and classification boundaries, see Carter et al., 2016.

\section{Support vector machine}

SVM classification algorithms were used to find the optimal solution to separate data into two different classes or categories. Briefly, SVMs function by plotting each input point in highdimensional space and defining the optimal hyperplane that best separates the data into classes. The hyperplane is defined by finding certain data points at or near class boundaries (support vectors) and maximizing the distance (margin) between each support vector and the line separating them (the hyperplane). These analyses employed a linear kernel or a linear hyperplane to predict functional decline as a binary outcome 24 months after a baseline assessment. Variables identified during the variable selection step were used as predictors. 
SVM classifiers were tuned for the optimum cost parameter prior to model evaluation in the testing sample. The cost parameter, $c$, is a regularization parameter, or the penalty for misclassification. The magnitude of $c$ affects the size of the margin-hyperplane and can be tuned for the optimal trade-off between training errors and testing errors to optimize the model for generalizability. For these analyses, we tested several values of $\mathrm{c}\left(2^{-8}, 2^{-4}, 2^{-2}, 1,2,16\right)$ to define the best model. The tuning process underwent cross-validation via repeated $\mathrm{k}$-fold crossvalidation.

For these analyses, k was set to 10 and 50 repeats were performed. This method of cross-validation works by randomly partitioning the sample into $10(\mathrm{k})$ equal-sized groups. The first nine groups are used to train a model with a given value of a tuning parameter. The tenth group is then used to evaluate this model. A new model is then trained on nine of the groups while a different group is used for testing. This process continues until each group is used for model evaluation exactly once. The entire process is repeated 50 times for each value of the tuning parameter. This information was then used to select the model tuning parameters which were used for the final model that was evaluated in the testing sample.

ROC curves were generated in the testing sample after variable selection and tuning in the training sample. Predictive ability of the models was assessed using AUC rather than accuracy because AUC has been shown to be a more consistent measure of evaluating models (Ling et al., 2003); an AUC of 0.5 indicates no discrimination, 0.5-0.6 indicates poor discrimination, 0.7 to 0.8 indicates acceptable discrimination, 0.8 to 0.9 indicates excellent discrimination, and greater than 0.9 indicates outstanding discrimination.

\section{Individual variable analysis}

\section{Variable selection}

To determine which individual variables best predicted functional decline, the 15 variables (out of the 508 extracted from ADNI) with the highest variable importance scores were selected 
and retained for additional statistical analyses. As with data modality variable selection, variable importance scores were calculated by defining a line that most accurately separated the two classes; ROC curves were generated and AUCs were calculated. Doing so allowed the identification of the most predictive variables independent of data modality.

\section{Statistical analysis}

Regression analysis was conducted using $\triangle \mathrm{FAQ}$ as a semi-continuous outcome variable and the 15 variables with the highest individual AUC included as predictors. $\triangle F A Q$ was calculated as the difference between the baseline FAQ score and 24-month FAQ score. Higher scores indicated worse functioning at the follow-up visit relative to the baseline visit. To circumvent issues of collinearity and minimize variance inflation factor (VIF) values, two variables were removed prior to regression analyses due to high correlations with other variables (maximum SUV of left angular gyrus, and EcogSP-total). Because the mean SUV in the left angular gyrus encompasses the maximum SUV as well as additional information about activity in this region, the maximum SUV in the left angular gyrus was removed. The EcogSP-total score is calculated as the average of all subtest scores. In order to include the most information, the EcogSP-total was removed. The left subiculum volume and left CA2/3 volume were also highly correlated. Rather than choose one to remove, these two values were added together for the regression analysis. Supplemental Fig. 1 reports the correlations between each of the 15 variables. For all statistical analyses, significance threshold was set at $p<0.05$.

\section{Data availability}

Data used for these analyses are available by request at adni.loni.usc.edu.

\section{Results}

\section{Demographic characteristics}

Table 1 reports demographic characteristics of the participant sample. In total, 398 participants (53.8\% male) between the ages of 55 and 90 (mean age $=71.4$; $\mathrm{SD}=6.9$ ) were included in 
these analyses. These participants were randomly partitioned into a training sample $(70 \%$; $\mathrm{N}=278)$ and a testing sample $(30 \% ; \mathrm{N}=120)$. The two groups did not differ significantly on age $(p=0.77)$, sex $(p=0.91)$, education $(p=0.74)$, baseline FAQ score $(p=0.30), \Delta$ FAQ score $(p=0.54)$, or proportion of participants with declining functioning $(p=1.00)$.

\section{Table 1}

Demographic information and comparisons between the testing and testing sample

\begin{tabular}{lllll}
\hline Variable & $\begin{array}{l}\text { All } \\
\text { Mean (SD) }\end{array}$ & $\begin{array}{l}\text { Training } \\
\text { Mean (SD) }\end{array}$ & $\begin{array}{l}\text { Testing } \\
\text { Mean (SD) }\end{array}$ & $\boldsymbol{p}$-value \\
\hline $\mathrm{N}$ & 398 & 278 & 120 & \\
Age & $71.4(6.9)$ & $71.3(6.7)$ & $71.5(7.4)$ & 0.77 \\
Males, N (\%) & $214(53.8)$ & $150(54.0)$ & $64(53.3)$ & 0.91 \\
Education & $16.5(2.5)$ & $16.5(2.5)$ & $16.4(2.5)$ & 0.74 \\
Diagnosis, N (\%) & & & & 0.94 \\
$\quad$ Cognitively Normal & $128(32.1)$ & $91(32.7)$ & $37(30.8)$ & \\
$\quad$ MCI & $270(67.8)$ & $187(67.3)$ & $83(69.2)$ & \\
Baseline FAQ & $1.9(3.4)$ & $1.8(3.3)$ & $2.1(3.6)$ & 0.30 \\
Declining Functioning, N (\%) & $133(33.4)$ & $93(33.5)$ & $40(33.3)$ & 1.00 \\
$\Delta$ FAQ & $1.4(4.1)$ & $1.6(4.4)$ & $1.1(3.8)$ & 0.54 \\
\hline
\end{tabular}

$\mathrm{a}=$ score between 0 and 30 , higher score indicates declining functioning

Abbreviations=FAQ=Functional Activities Questionnaire; $\mathrm{SD}=$ standard deviation; $\mathrm{MCI}=$ mild cognitive impairment

\section{Most predictive data modality}

Table 2 reports the variables with greatest AUC that were selected from each modality; Table 3 and Fig. 2 report model performance and confidence intervals of these variables in the testing sample. AUC ranged from poor (0.62) to acceptable (0.77). The best single-modality accuracy was achieved with neurocognitive measurements (Fig. 2a). This model demonstrated acceptable AUC $(0.77,95 \% \mathrm{CI}=0.68-0.86)$ and accuracy $(74.2 \%)$. MRI metrics, FDG-PET metrics, genetic/fluid-based biomarkers, and demographic information all demonstrated poor AUC, 0.62, 0.66, 0.63, and 0.62 respectively (Fig 2d, 2b, 2c, Fig 2e). 
Table 2

Top ranked variables of each data modality as measured via individual AUC

\begin{tabular}{|c|c|c|c|c|c|}
\hline Rank & Demographics $^{\text {a }}$ & MRI $^{\mathbf{a}}$ & FDG-PET $^{b}$ & Neurocognitive $^{b}$ & Biomarkers $^{b}$ \\
\hline 1 & $\begin{array}{l}\text { Diagnosis (MCI or } \\
\mathrm{CN})\end{array}$ & $\begin{array}{l}\text { R hippocampus } \\
\text { volume }\end{array}$ & $\begin{array}{l}\text { Maximum } \mathrm{L} \\
\text { angular gyrus }\end{array}$ & CDR-sum of boxes & Amyloid beta \\
\hline 2 & $\begin{array}{l}\text { Psychiatric } \\
\text { diagnosis }\end{array}$ & L CA2/3 volume & $\begin{array}{l}\text { Mean L angular } \\
\text { gyrus }\end{array}$ & EcogSP-Total & Albumin \\
\hline 3 & $\begin{array}{l}\text { Dermatological } \\
\text { diagnosis }\end{array}$ & $\begin{array}{l}\text { L subiculum } \\
\text { volume }\end{array}$ & $\begin{array}{l}\text { Median L angular } \\
\text { gyrus }\end{array}$ & $\begin{array}{l}\text { Logical memory } \\
\text { delayed }\end{array}$ & Tau \\
\hline 4 & $\begin{array}{l}\text { Gastrointestinal } \\
\text { diagnosis }\end{array}$ & $\begin{array}{l}\mathrm{L} \mathrm{CA} 4 / \mathrm{DG} \\
\text { volume }\end{array}$ & $\begin{array}{l}\text { Maximum BL } \\
\text { cingulum post }\end{array}$ & EcogSP-Memory & p-tau \\
\hline 5 & Malignancy & R CA2/3 volume & $\begin{array}{l}\text { Max R angular } \\
\text { gyrus }\end{array}$ & $\begin{array}{l}\text { EcogSP-Divided } \\
\text { Attention }\end{array}$ & PHS \\
\hline 6 & $\begin{array}{l}\text { Musculoskeletal } \\
\text { diagnosis }\end{array}$ & $\begin{array}{l}\text { R CA4/DG } \\
\text { volume }\end{array}$ & $\begin{array}{l}\text { Mean R angular } \\
\text { gyrus }\end{array}$ & ADAS13 & Total protein \\
\hline 7 & Cardiac diagnosis & $\begin{array}{l}\text { L hippocampus } \\
\text { volume }\end{array}$ & $\begin{array}{l}\text { Median BL } \\
\text { cingulum post }\end{array}$ & MoCA & $\begin{array}{l}\text { Percent } \\
\text { eosinophils }\end{array}$ \\
\hline 8 & Age & $\begin{array}{l}\text { R subiculum } \\
\text { volume }\end{array}$ & $\begin{array}{l}\text { Mode R angular } \\
\text { gyrus }\end{array}$ & EcogSP-Language & Neutrophils \\
\hline 9 & Prior surgery & $\begin{array}{l}\text { L entorhinal } \\
\text { cortical thickness }\end{array}$ & $\begin{array}{l}\text { Median R angular } \\
\text { gyrus }\end{array}$ & $\begin{array}{l}\text { RAVLT 30-min } \\
\text { delay }\end{array}$ & $\begin{array}{l}\text { Percent } \\
\text { neutrophils }\end{array}$ \\
\hline 10 & Education & $\begin{array}{l}\text { L entorhinal } \\
\text { cortical volume }\end{array}$ & $\begin{array}{l}\text { Minimum } \mathrm{R} \\
\text { angular gyrus }\end{array}$ & $\begin{array}{l}\text { Category fluency- } \\
\text { animals }\end{array}$ & Serum glucose \\
\hline 11 & $\begin{array}{l}\text { Endocrine/metabolic } \\
\text { diagnosis }\end{array}$ & R CA1 volume & $\begin{array}{l}\text { Maximum L } \\
\text { temporal gyrus }\end{array}$ & $\begin{array}{l}\text { RAVLT \% } \\
\text { forgetting }\end{array}$ & Eosinophils \\
\hline 12 & Smoking status & $\begin{array}{l}\mathrm{R} \text { entorhinal } \\
\text { cortical thickness }\end{array}$ & $\begin{array}{l}\text { Mean BL } \\
\text { cingulum post }\end{array}$ & $\begin{array}{l}\text { EcogSP- } \\
\text { Visuospatial }\end{array}$ & $\begin{array}{l}\text { Percent } \\
\text { lymphocytes }\end{array}$ \\
\hline 13 & $\begin{array}{l}\text { Hematopoietic- } \\
\text { Lymphatic } \\
\text { diagnosis }\end{array}$ & $\begin{array}{l}\mathrm{R} \text { hippocampal tail } \\
\text { volume }\end{array}$ & $\begin{array}{l}\text { Median L } \\
\text { temporal gyrus }\end{array}$ & RAVLT Trial 3 & Basophils \\
\hline 14 & $\begin{array}{l}\text { Neurological } \\
\text { diagnosis (Not AD) }\end{array}$ & L CA1 volume & $\begin{array}{l}\text { Mean L temporal } \\
\text { gyrus }\end{array}$ & $\begin{array}{l}\text { RAVLT } \\
\text { immediate }\end{array}$ & $\begin{array}{l}\text { Gamma-glutamyl } \\
\text { Transferase }\end{array}$ \\
\hline 15 & Gender & $\begin{array}{l}\text { L Presubiculum } \\
\text { volume }\end{array}$ & $\begin{array}{l}\text { Mean R temporal } \\
\text { gyrus }\end{array}$ & RAVLT Trial 5 & Percent monocytes \\
\hline
\end{tabular}

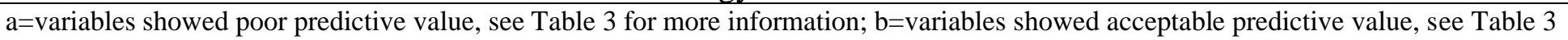
for more information

Abbreviations; $\mathrm{AD}=\mathrm{Alzheimer}$ 's disease; $\mathrm{ADAS}=\mathrm{Alzheimer}$ 's Disease Assessment Scale; $\mathrm{BMI}=$ body mass index; $\mathrm{CDR}=\mathrm{Clinical}$ Dementia Rating; CN=Cognitively normal; DG=Dentate gyrus; EcogSP=Everyday Cognition-Study Partner; ENT=Ears/Nose/Throat; FDG=fluorodeoxyglucose; L=left; MCI=mild cognitive impairment; MoCA=Montreal Cognitive Assessment; PHS=polygenic hazard score; -tau=phosphorylated tau; R=right; RAVLT=Rey Auditory Verbal Learning Test

Table 3

\begin{tabular}{lcccccc}
\hline Model & $\begin{array}{c}\text { Sensitivity } \\
\text { \% }\end{array}$ & $\begin{array}{c}\text { Specificity } \\
\text { \% }\end{array}$ & Accuracy(\%) & AUC & AUC & C \\
\hline Neurocognitive & 0.48 & 0.88 & 74.2 & 0.77 & $0.68-0.86$ & $2^{-8}$ \\
FDG-PET & 0.33 & 0.90 & 70.8 & 0.66 & $0.56-0.77$ & $2^{-2}$ \\
Genetic/fluid-based biomarkers & 0.23 & 0.95 & 70.8 & 0.63 & $0.51-0.74$ & $2^{-4}$ \\
MRI & 25.0 & 95.0 & 71.7 & 0.62 & $0.51-0.73$ & $2^{0}$ \\
Demographics & 0.00 & 100.0 & 66.7 & 0.62 & $0.51-0.72$ & $2^{-8}$ \\
\hline
\end{tabular}

Abbreviations: $\mathrm{AUC}=$ area under the curve; $\mathrm{FDG}=$ fluorodeoxyglucose 
Fig. 2. Classifier results
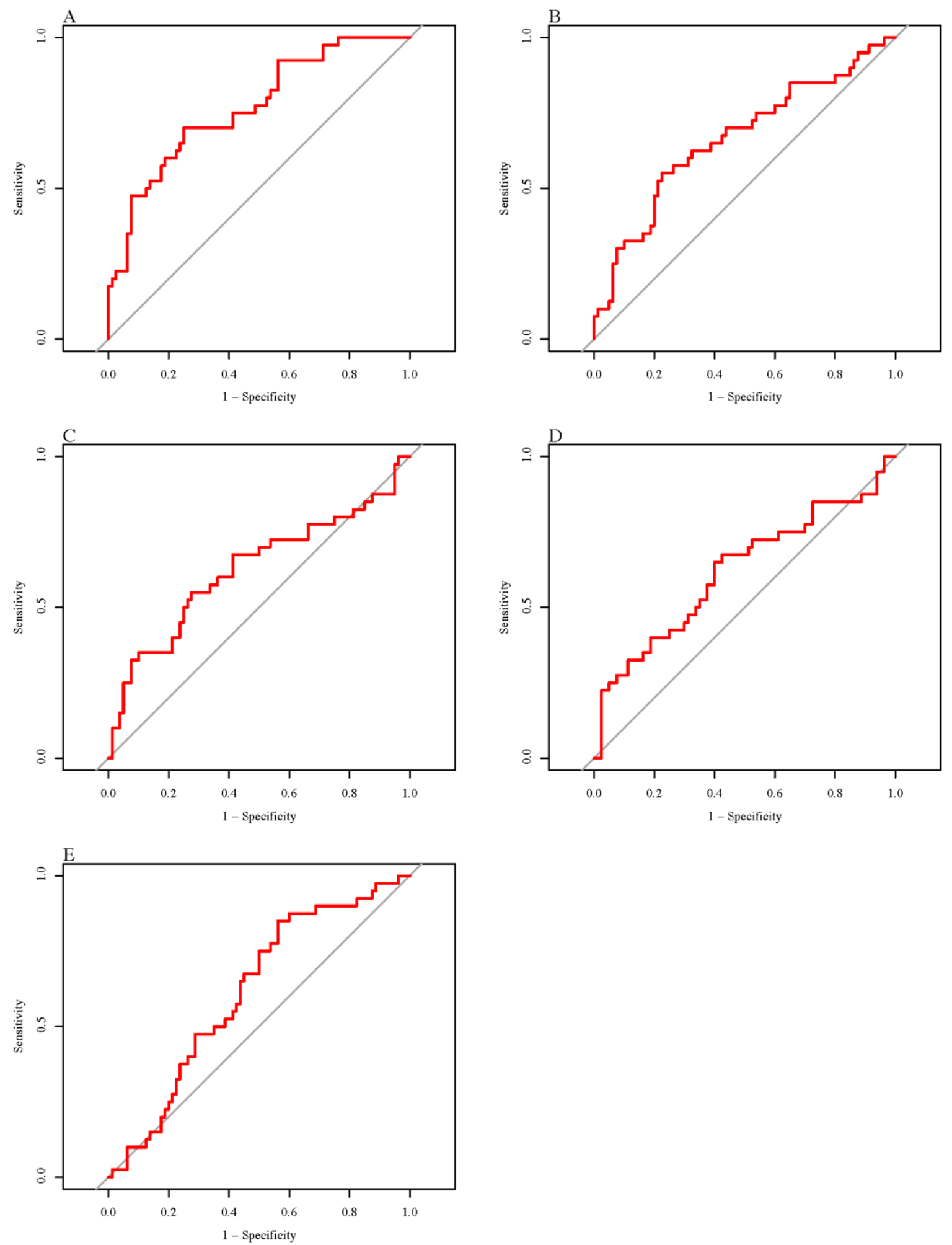

A Neurocognitive measures

B FDG-PET measures

C Genetics/fluid-based biomarkers

D MRI measures 
Predicting functional decline

E Demographic information

Abbreviations: FDG=fluorodeoxyglucose

\section{Most predictive variable}

The 15 variables with the highest individual AUCs from all 508 variables, listed alphabetically, were: Alzheimer's Disease Assessment Scale (ADAS13), Category fluency-animals, Clinical Dementia Rating (CDR)-sum of boxes, Everyday Cognition-Study Partner (EcogSP)-Divided Attention, EcogSP-Language, EcogSP-Memory, EcogSP-Total, EcogSP-Visuospatial, left CA2/CA3 volume, left subiculum volume, Logical Delayed Memory, mean SUV of left angular gyrus, maximum SUV of left angular gyrus, Rey Auditory Verbal Learning Test (RAVLT) 30-minute delay, right hippocampal volume, and the total Montreal Cognitive Assessment (MoCA) score. These variables (with the exception of maximum SUV of the left angular gyrus and EcogSP-total; see above were then entered into a multiple regression model in the testing sample to predict $\triangle \mathrm{FAQ}$ as a semi-continuous variable. Basic demographic variables were not added as covariates because they were not identified as significant predictors during variable selection.

Because the models demonstrated heteroskedasticity, a weighting parameter was used such that the absolute value of the residuals was regressed against each predictor. This improved heteroskedasticity. Furthermore, all models described met assumptions of a linear regression: the mean of the residual values was approximately zero; there was no autocorrelation of residuals. predictors and residuals showed no significant correlation; the predictors all showed positive variance; there was no multicollinearity present; and the residuals values were approximately normally distributed. Additionally, because weighting parameters tend to lead to inflated $\mathrm{R}^{2}$ values, all reported $\mathrm{R}^{2}$ values have been adjusted as per Willet and Singer (1988). This adjusted calculates $\mathrm{R}^{2}$ based on the difference between the model estimates and the original values and had been shown to be a less biased goodness of 
Predicting functional decline

fit. Lastly, the final model included 12 predictors for 120 participants, which is in line with the recommendation of 1:10 predictor to participant ration for regression analysis (Peduzzi et al., 1995, 1996).

The overall regression model was significant $\left(F(12,107)=16.69, p<2.2 \times 10^{-16}\right.$ and explained $33.1 \%$ of the variance in functional change (Table 4). In this model, only EcogSPLanguage, mean left angular gyrus activity, and ADAS13 were significant predictors. Those who scored poorly on EcogSP-Language showed greater decline in functioning at the 24-month follow-up $\left(F(1,118)=71.81, p=7.8 \times 10^{-4}\right.$; Fig. 3a). The EcogSP-Language subtest explained $21.1 \%$ of the variance in functional change. Those with lower mean activity in the left angular gyrus also showed greater decline at the 24-month follow-up $\left(F(1,118)=58.66, p=5.8 \times 10^{-12}\right.$; Fig. 3b). Left angular gyrus activity explained $14.5 \%$ of the variance in functional change. Those who scored poorly on the ADAS13 also showed greater decline in functioning at the 24month follow-up $\left(F(1,118)=1237, p<2.2 \times 10^{-16}\right.$; Fig. 3c). The ADAS13 explained $21.3 \%$ of the variance in functional change. After accounting for shared variance, these three variables collectively explained $31.7 \%$ of the total variance in functional decline $(F(2,116)=74.56, p<$ $\left.2.2 \times 10^{-16}\right)$. 
Predicting functional decline

\section{Table 4}

Results of Multiple Regression

\begin{tabular}{llllll}
\hline Variable & Coefficient & $\boldsymbol{S E}$ & $\mathbf{9 5 \%}$ CI & VIF & $\boldsymbol{p}$-value \\
\hline ADAS13 & 0.15 & 0.03 & $0.09-0.21$ & 3.13 & $8.2 \times 10^{-6^{*}}$ \\
Category fluency-animals & -0.04 & 0.03 & $-0.10-0.01$ & 1.56 & 0.18 \\
CDR-Sum of boxes & -0.10 & 0.14 & $-0.38-0.19$ & 1.64 & 0.50 \\
EcogSP-Divided Attention & -0.46 & 0.26 & $-0.98-0.05$ & 2.85 & 0.08 \\
EcogSP-Language & 1.55 & 0.31 & $0.93-2.17$ & 3.00 & $2.6 \times 10^{-6^{*}}$ \\
EcogSP-Memory & 0.18 & 0.30 & $-0.41-0.77$ & 3.83 & 0.55 \\
Mean Left Angular Gyrus & -3.05 & 0.73 & $-4.49--1.60$ & 1.34 & $6.28 \times 10^{-5 *}$ \\
Left CA2/3 + subiculum & 0.00 & 0.00 & $-0.001-0.001$ & 4.05 & 0.63 \\
Logical Memory Delayed & 0.03 & 0.03 & $-0.03-0.09$ & 2.09 & 0.30 \\
MoCA & 0.02 & 0.06 & $-0.09-0.14$ & 2.87 & 0.68 \\
Right Hippocampal Volume & 0.00 & 0.00 & $-0.001-0.00$ & 4.30 & 0.55 \\
RAVLT 30min Delay & 0.04 & 0.03 & $-0.02-0.10$ & 1.98 & 0.18 \\
Overall Model & $0.331^{\mathrm{a}}$ & & $16.69^{\mathrm{b}}$ & & $<2.2 \times 10^{-16^{*}}$ \\
\hline a=Model R ${ }^{2}$; b=F-statistic; * indicates p<0.05 & \multicolumn{3}{|}{} \\
Abbreviations: ADAS13=Alzheimer's Disease Assessment & Schedule; CDR=Clinical Dementia Rating; \\
EcogSP=Everyday Cognition-Study Partner; MoCA=Montreal Cognitive Assessment; RAVLT=Rey Auditory \\
Verbal Learning Test; SE=standard error; VIF-variance inflation factor & &
\end{tabular}


Fig. 3. Relationship between significant predictors and $\triangle F A Q$
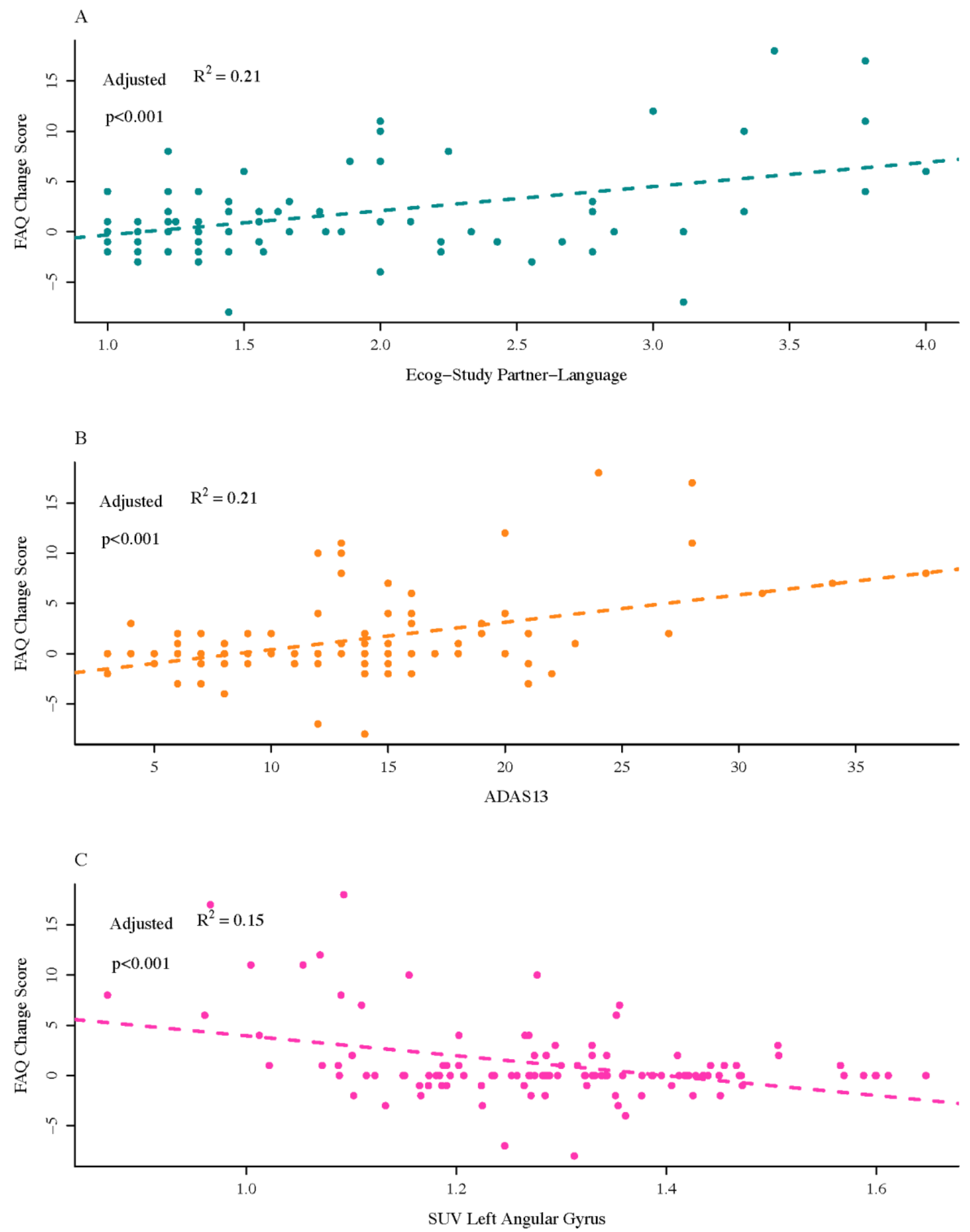

A Values on the $\mathrm{x}$-axis represent study partner report for language ability on the Everyday Cognition assessment, with higher scores indicating worse language ability. Scores are calculated as the average of the 9 items on the subtest. Values on the y-axis represent $\triangle F A Q$, calculated as the difference between baseline FAQ score and 24-month FAQ score, with higher scores indicating worse functioning as the 24-month visit. Worse partner-reported language ability was associated with greater decline in functioning 24 months after baseline assessment. 
B Values on the $\mathrm{X}$-axis represent mean SUV of the left angular gyrus, with lower scores indicating lower brain activity. Values on the $y$-axis represent $\triangle F A Q$, calculated as the difference between baseline FAQ score and 24-month FAQ score, with higher scores indicating worse functioning at the 24-month visit. Lower average glucose metabolism in the left angular gyrus was associated with greater decline in functioning 24 months after baseline assessment.

$\mathrm{C}$ Values on the $\mathrm{x}$-axis represent ADAS13 scores, with higher scores indicating worse performance. Values on the $\mathrm{y}$-axis represent $\triangle \mathrm{FAQ}$, calculated as the difference between baseline FAQ score and 24-month FAQ score, with higher scores indicating worse functioning at the 24-month visit. Worse ADAS13 scores were associated with greater decline in functioning 24 months after baseline assessment.

Abbreviations: EcogSP=Everyday Cognition-Study Partner; FAQ=Functional Activities Questionnaire; SUV=standardized uptake value

\section{Discussion}

The objective of this study was to use machine learning to reliably predict functional decline within 24 months in cognitively healthy older adults and those with MCI. We first examined clinically relevant modalities (i.e., demographics, MRI, FDG-PET, neurocognitive measures, and genetic/fluid-based biomarkers) that predicted functional decline, and then examined individual variables across all modalities. There were two main findings. First, neurocognitive measures demonstrated the best accuracy in predicting functional decline compared to other data modalities $($ accuracy $=74.2 \%$; AUC $=0.77)$, followed by FDG-PET $($ accuracy $=70.8 \%$; AUC $=0.66$ ). Second, when considering the top 15 variables selected by the training sample, only scores on the EcogSP-Language subtest, ADAS13, and mean activity in the left angular gyrus (measured by FDG-PET) explained a significant amount of variance in functional decline in the testing sample.

Results from the SVM algorithms demonstrated that neurocognitive measures predict IADL decline in two years with acceptable discriminability and outperform other measures including neuroimaging, CSF, demographics, and genetics. These results suggest that neurocognitive tests can successfully predict functional decline better than more invasive and costly assessments. Moreover, when examining individual variables' discriminatory ability across all modalities, 11 of the top 15 predictive variables were neurocognitive. Of these neurocognitive measures, the EcogSP-language subtest and the ADAS13 demonstrated the 
strongest association with functional decline in the testing sample. The EcogSP-language subtest specifically explained $21.1 \%$ of the variance. This is consistent with similar work that has identified the Ecog as a predictor of cognitive decline and conversion to MCI or Alzheimer's disease (Nosheny et al., 2019). The Ecog is an informant-report questionnaire designed to assess subtle changes in real-world functioning in an older population; it can also be administered as a self-report measure. It has shown high test-retest reliability $(r=0.82)$, convergent validity with widely used neuropsychological and informant assessments, and external validity in discriminating normal, MCI, and dementia groups (Farias et al., 2008). The EcogSP-language subtest includes nine items that assess language and semantic memory (Farias et al., 2008). However, it is likely that the EcogSP-language subtest assesses more complex cognitive abilities than simple language. For example, understanding and communicating in a conversation requires understanding grammatical complexities of language (semantic memory), following and remembering the sequence of remarks made by various speakers (working memory and attention), and retrieving specific event memories to convey thoughts (episodic memory), which decline with age (Salthouse, 2010; Rabaglia and Salthouse, 2011; Rudner and Signoret, 2016). Traditional language fluency and verbal knowledge tests such as the Category Fluency Test and the American National Adult Reading Test (ANART) were not selected as significant predictors of functional decline in the present study, suggesting that EcogSP-language subtest may be probing more complex information synthesis and processing abilities that underlie functional ability. The Ecog-language subtest as completed by the study participant also was not selected as a significant predictor. It is possible that a key feature of this assessment in predicting functional decline is in the study partner's assessment of such skills, as study participants may be anosognosic. This is supported by other research that suggests that informant-reported ratings of functional deficits better predict decline and conversion to dementia (Tabert et al., 2002). 
The ADAS13 also demonstrated a significant $\left(p<2.2 \times 10^{-16}\right)$ association with functional decline and explained $21.3 \%$ of the variance in decline. The ADAS13 is a cognitive test (scores between 0 and 85 with higher scores indicating worse cognition) that assesses patients in various domains including learning and memory, language production and comprehension, and constructional and ideational praxis. It is derived from the ADAS11 which was designed to help diagnose mild and moderate Alzheimer's disease (Rosen et al, 1984), and incorporates two additional items (cancellation and delayed free recall) that have been shown to increase the sensitivity of the assessment in differentiating between $\mathrm{CN}, \mathrm{MCI}$, and $\mathrm{AD}$ (Mohs et al, 1997; Sano et al, 2011). While this newer version of the ADAS has not yet been well-studied, it has been shown to be predictive of conversion from MCI to Alzheimer's disease (Zhou et al., 2012), help determine amyloid $\beta$ positivity (Ba et al., 2019), and identify early stages of Alzheimer's disease (Albright J. 2019). Because the ADAS13 is made of multiple domains, it is possible that the results here are being driven by one or several specific areas of cognition. One study showed that five domains were particularly sensitive to cognitive change over time - commands, remember instructions, comprehension, word finding, and spoken language (Dowling et al., 2016). Our results suggest that the ADAS13 can be a good measure to understand functional decline and the relevant domains may be similar to the construct uncovered by our results. Future works may help determine the importance and relevance of this assessment and potential construct.

Following neurocognitive measures, FDG-PET demonstrated the next best discriminability $(\mathrm{AUC}=0.66)$. FDG-PET has long been used to assess cognitive decline and dementia by serving as a proxy for brain activity. A previous study demonstrated the importance of FDG-PET in predicting functional decline in individuals with MCI (Landau et al., 2011). While Landau and colleagues used a composite measure of the five ROIs also used in this study, the results here specifically identified the left angular gyrus as a significant 
predictor of functional decline. Further validating the angular gyrus results, the left angular gyrus was a significant predictor in a separate sample that had not been used for variable selection. Regression analysis indicated that activity in the left angular gyrus alone explained $14.5 \%$ of the variance in degree of functional change, which underscores the importance of considering the left angular gyrus to develop a more comprehensive understanding of functional decline throughout the aging process.

The angular gyrus resides in the posterior inferior parietal lobe in Brodmann area 39 and is part of the parietal association cortex (Cabeza et al., 2012; Seghier, 2013). It serves as a hub linking large scale neural networks, including the default mode network, dorsal attention network, and visual and somatosensory networks, suggesting that this area is critically involved in synthesizing heteromodal information (Tomasi and Volkow, 2011). In a review integrating a large body of primary research, Seghier and colleagues (2013) conclude that "the [angular gyrus] resembles a cross-modal integrative hub that gives sense and meaning to an event within a contextualized environment, based on prior expectations and knowledge, and toward an intended action" (p. 52). This observation comes from studies supporting the angular gyrus's role in semantic associations (Vigneau et al., 2006), reading and comprehension (Houdé et al., 2010), episodic memory retrieval (Hutchinson et al., 2009; Kalpouzos et al., 2010; Hayes et al., 2011), verbal working memory (Emch et al., 2019), and social cognition (Mar, 2011). The left angular gyrus may play a more substantial role in semantic and conceptual knowledge relative to the right angular gyrus (Cabeza et al., 2012). Moreover, degeneration specifically in the left angular gyrus has been associated with cognitive decline in neurodegenerative disease (Mihaescu et al., 2019). It is plausible that the ability to carry out IADLs, which depends on the nexus of conceptual knowledge and action sequences, critically relies on left angular gyrus function. The results reported here are consistent with the notion that disruptions in IADLs reflect lower brain activation in this area. While a previous study has demonstrated 
a cross-sectional link between angular gyrus volume and functional ability (Griffith et al., 2010), the present study provides evidence for the association between angular gyrus function and future functional decline.

Of the variables identified in the training sample and validated in the testing sample, processing, retrieval, and conceptual integration of semantic information emerged as a construct that may be critical for understanding risk factors of future functional decline. This construct is indicated by the EcogSP-Lang and mean SUV in the left angular gyrus. These two measures, one neurocognitive and one neurometabolic, overlap in their purported involvement in integration of conceptual knowledge and action sequences toward a functional goal. The ADAS13 also incorporates semantic language processing items, and future research should examine if these particular items, such as those identified in Dowling et al. (2016) may be driving this relationship.

Results from the SVM models also show that genetic/fluid-based biomarkers and MRI metrics demonstrate minimal predictive ability, with an AUC of 0.63 and 0.62, respectively. This provides some support for prior research demonstrating their ability to predict functional decline in adults with normal cognition and mild cognitive impairment (Okonkwo et al., 2010a, b). However, when the top 15 variables were validated in an independent sample, no genetic/fluid-based biomarkers or MRI metrics were selected, suggesting that while they may demonstrate predictive ability, this predictive ability may be outranked when other variables are considered. This underscores the importance of considering a large set of variables in a data-driven approach in order to identify those that are most predictive compared to selecting variables a priori. While demographic information demonstrated some predictive ability as measured by AUC (0.62), it showed 0\% sensitivity, suggesting such variables have minimal utility for studying functional decline over two years. 
This study has several limitations. First, it is important to consider potential biases in sample selection. Participants included in ADNI are, on average, highly educated and predominantly white. Socioeconomic status has been shown to affect IADL functional status (Seeman et al., 2010). Therefore, these results may not generalize to the larger sociocultural context that is representative of individuals within the United States. The present study also only examines the predictive ability of various data modalities over 24 months. It cannot be determined whether other data modalities would show greater predictive ability at different timepoints relative to functional decline. Further, these analyses only included data that were already processed through ADNI. Other modalities, such as functional MRI (fMRI), might improve model performance and predictive power.

It is worth noting that sensitivity in this sample is low. This pattern may be attributed to the multidetermined nature of functional status and decline. Functional ability may be impacted by a number of different factors, including neurodegeneration, physical impairment, and medical illness-induced limitations. While we have tried to include a number of diverse variables in the model, it may not be feasible to capture their later effects with baseline variables. For example, an unexpected stroke could severely affect functional decline, but this may not be indicated by any baseline health variable. Additionally, slow physical decline due to illness such as arthritis may impact certain IADL domains such as shopping alone or traveling out of the neighborhood, but this slow decline may be not evident by baseline status; also, such an illness may not have impacted enough people in the model to affect variable selection. Moreover, research has also demonstrated that the greater the time difference between assessment and prediction, the lower the sensitivity of the model (Ritter et al., 2015). Presently, the models developed would require additional information and further validation to improve sensitivity before model deployment in a clinical setting. Nonetheless, the patterns 
uncovered here shed important light on variables, modalities, and constructs that contribute to our understanding of functional decline and can help guide future research.

\section{Conclusions}

The machine learning approach applied here improves upon previous work examining the ability of neuroimaging, neurocognitive, demographic, and genetic/fluid-based biomarkers to predict functional decline two years after baseline assessment. Neurocognitive measures showed the highest accuracy and best discriminative ability, suggesting that a set of inexpensive and non-invasive cognitive assessments can be used to predict independent functioning in lieu of expensive and more invasive measures. Our data-driven approach identified three novel measures (EcogSP-Language, ADAS13, and mean left angular gyrus activity) to predict functional decline, which had not been reported when selecting variables $a$ priori or when looking at smaller subsets of variables. Two of these markers (left angular gyrus activity and EcogSP-Language) are purported to be involved in the processing, retrieval, and conceptual integration of semantic information, highlighting this construct in functional ability. The third marker (ADAS13) is a global measure of cognition that may be uniquely designed to target functional decline and certain domains assessed with this tool may be related to the above construct. Future work should consider the utility of the EcogSP-Language, a simple informant report questionnaire, for predicting functional decline in the absence of more invasive procedures such as PET imaging or CSF markers or longer neuropsychological assessments. This questionnaire is non-invasive, time-limited, and cost effective, and the results reported here suggest it may be a useful prediction tool. Future work should further consider the role of the angular gyrus in functional decline in aging and neurodegenerative disease.

\section{Acknowledgements}


Predicting functional decline

The authors graciously acknowledge the support and participation from all those involved in the Alzheimer's Disease Neuroimaging Initiative.

\section{Funding}

This work was supported by the National Institute on Aging (NIA) of the National Institutes of Health (NIH) R01AG058822 (awarded to JPH), R21AG056921 (awarded to SMH), and The Ohio State University Discovery Themes Chronic Brain Injury Initiative (JPH and SMH). The content is solely the responsibility of the authors and does not necessarily represent the official views of the NIH.

Data collection and sharing for this project was funded by the Alzheimer's Disease Neuroimaging Initiative (ADNI) (NIH Grant U01 AG024904) and DOD ADNI (Department of Defense award number W81XWH-12-2-0012). ADNI is funded by the NIA, the National Institute of Biomedical Imaging and Bioengineering, and through generous contributions from the following: AbbVie, Alzheimer's Association; Alzheimer's Drug Discovery Foundation; Araclon Biotech; BioClinica, Inc.; Biogen; Bristol-Myers Squibb Company; CereSpir, Inc.; Cogstate; Eisai Inc.; Elan Pharmaceuticals, Inc.; Eli Lilly and Company; EuroImmun; F. Hoffmann-La Roche Ltd and its affiliated company Genentech, Inc.; Fujirebio; GE Healthcare; IXICO Ltd.; Janssen Alzheimer Immunotherapy Research \& Development, LLC.; Johnson \& Johnson Pharmaceutical Research \& Development LLC.; Lumosity; Lundbeck; Merck \& Co., Inc.;Meso Scale Diagnostics, LLC.; NeuroRx Research; Neurotrack Technologies; Novartis Pharmaceuticals Corporation; Pfizer Inc.; Piramal Imaging; Servier; Takeda Pharmaceutical Company; and Transition Therapeutics. The Canadian Institutes of Health Research is providing funds to support ADNI clinical sites in Canada. Private sector contributions are facilitated by the Foundation for the NIH (www.fnih.org). The grantee organization is the Northern California Institute for Research and Education, and the study is coordinated by the Alzheimer's Therapeutic Research Institute at the University of Southern California. ADNI 
Predicting functional decline

data are disseminated by the Laboratory for Neuro Imaging at the University of Southern California.

\section{Competing interests}

None. 


\section{Supplementary Material}

\section{Materials and Methods}

\section{MRI acquisition and processing}

MR images were pre-processed by Mayo Clinic. The University of California San Francisco performed automated cortical reconstruction and volumetric segmentation with FreeSurfer image analysis suite, version 5.1 available for download at (http://surfer.nmr.mgh.harvard.edu/). Scans were processed cross-sectionally using the 2010 Desikan-Killany atlas. The technical details of these procedures are described in prior publications (Dale and Sereno, 1993; Dale et al., 1999, Fischl et al., 1999a, b, 2001, 2002, 2004b, a; Fischl and Dale, 2000; Segonne et al., 2004; Han et al., 2006; Jovicich et al., 2006; Reuter et al., 2010, 2012). Briefly, this processing includes motion correction and averaging (Reuter et al., 2010) of multiple volumetric T1 weighted imagines (when more than one is available), removal of non-brain tissue using a hybrid watershed/surface deformation procedure (Segonne et al., 2004), automated Talairach transformation, segmentation of the subcortical white matter and deep gray matter volumetric structures (including hippocampus, amygdala, caudate, putamen, ventricles) (Fischl et al., 2002, 2004a), insensitivity normalization (Sled et al., 1998), tessellation of the gray matter white matter boundary, automated topology correction (Fischl et al., 2001; Segonne et al., 2007), and surface deformation following intensity gradients to optimally place the gray/white and gray/cerebrospinal fluid borders at the location where the greatest shift in intensity defines the transition to the other tissue class (Dale and Sereno, 1993; Dale et al., 1999; Fischl and Dale, 2000).

In order to ensure that high quality data MRI data was collected across sites, all MR images underwent image quality control at the central MRI core laboratory at Mayo Clinic, Rochester Minnesota. For ADNI-GO visits, a phantom scan was collected each day participants 
were scanned. These scans were checked to ensure proper scanner calibration and for detection of scanner changes indicative of underlying scanner issues. These scans were then used to identify and correct for change in geometric scaling over time, scanner qualification, scanner recalibration, and ongoing scanner quality (Gunter et al., 2009). For more information, description of the ADNI-GO MRI procedures may be found at http://www.adni-info.org. For ADNI2 visits, the phantom scan was used to certify and update scanners but was no longer collected each day. Improved vendor products were shown to address most of the artifacts that initially warranted a phantom scan. Further, they showed that consistent results could be achieved across different scanners after ADNI 1 (Jack et al., 2010).

Fluorodeoxyglucose-PET (FDG-PET)

Description of FDG-PET acquisition may be found at http://www.adni-info.org. Briefly, patients fasted for at least four hours prior to being injected with $\sim 185 \mathrm{MBq}$ of tracer and remained in a dim room for 20-30 minutes following injection. Dynamic 3D scans of six 5minute frames were collected between 30- and 60-minutes. All PET images underwent quality control checking at the University of Michigan. Images were co-registered to native space, averaged together, aligned, and smoothed to $8 \mathrm{~mm}$ resolution, which was the lowest resolution of any scanner used to collect data.

\section{Biological samples}

To calculate a polygenic hazard score (PHS) for Alzheimer's disease, single nucleotide polymorphisms (SNPs) were identified from a genome-wide association study in the International Genomics of Alzheimer's project (at $p<10^{-5}$ ). 31 SNPs and two APOE variants were integrated to create a single hazard score. This score predicts progression to Alzheimer's disease diagnosis as well as age of onset. For complete description of and methods used to calculate PHS, see (Desikan et al., 2017). 
Analyses of cerebrospinal fluid were performed at the UPenn/ADNI Biomarker Laboratory using Roche Elecsys immunoassay and following Roche Study protocol. The A $\beta$ CSF immunoassay has an upper limit of $1700 \mathrm{pg} / \mathrm{mL}$ and lower limit and 200pg/mL; beyond these limits, performance has not been established. Participants with $A \beta$ levels greater than the upper technical limit were truncated to $1700 \mathrm{pg} / \mathrm{mL}$. There were no individuals below the lower technical limit for $\mathrm{A} \beta$ or outside of the technical limits for tau $(80-1300 \mathrm{pg} / \mathrm{mL})$ or $\mathrm{p}$-tau $(8$ $120 \mathrm{pg} / \mathrm{mL})$.

\section{Supplemental Table 1}

Neurocognitive tests administered

\begin{tabular}{lll}
\hline Domain & Test & Citation \\
\hline General cognition & Montreal Cognitive Assessment (MOCA) & (Nasreddine et al., 2005) \\
& Alzheimer's Disease Assessment Scale-Cognitive & (Rosen et al., 1984) \\
& (ADAS-13) & \\
& Clinical Dementia Rating Scale (CDR) & (Berg, 1988) \\
& Everyday Cognition (Ecog) & (Farias et al., 2008) \\
& Mini-Mental Status Examination (MMSE) & (Folstein et al., 1975) \\
Executive function & Category Fluency Test & (Rosen, 1980) \\
& Trail Making Test-Part B & (Reitan, 1958) \\
& Clock Drawing Test & (Goodglass and Kaplan, 1982) \\
Learning and memory & Cognitive Change Index & (Saykin et al., 2006) \\
& Logical Memory Test I and II-delayed paragraph & (Wechsler, 1987) \\
& recall & \\
Language & Rey Auditory Verbal Learning Test (RAVLT) & (Rey, 1941) \\
& Boston Naming Test & (Kaplan et al., 1983) \\
Processing speed & American National Adult Reading Test & (Nelson and O'Connell, 1978) \\
\hline
\end{tabular}




\section{Supplement Fig. 1. Correlation plot of selected variables}

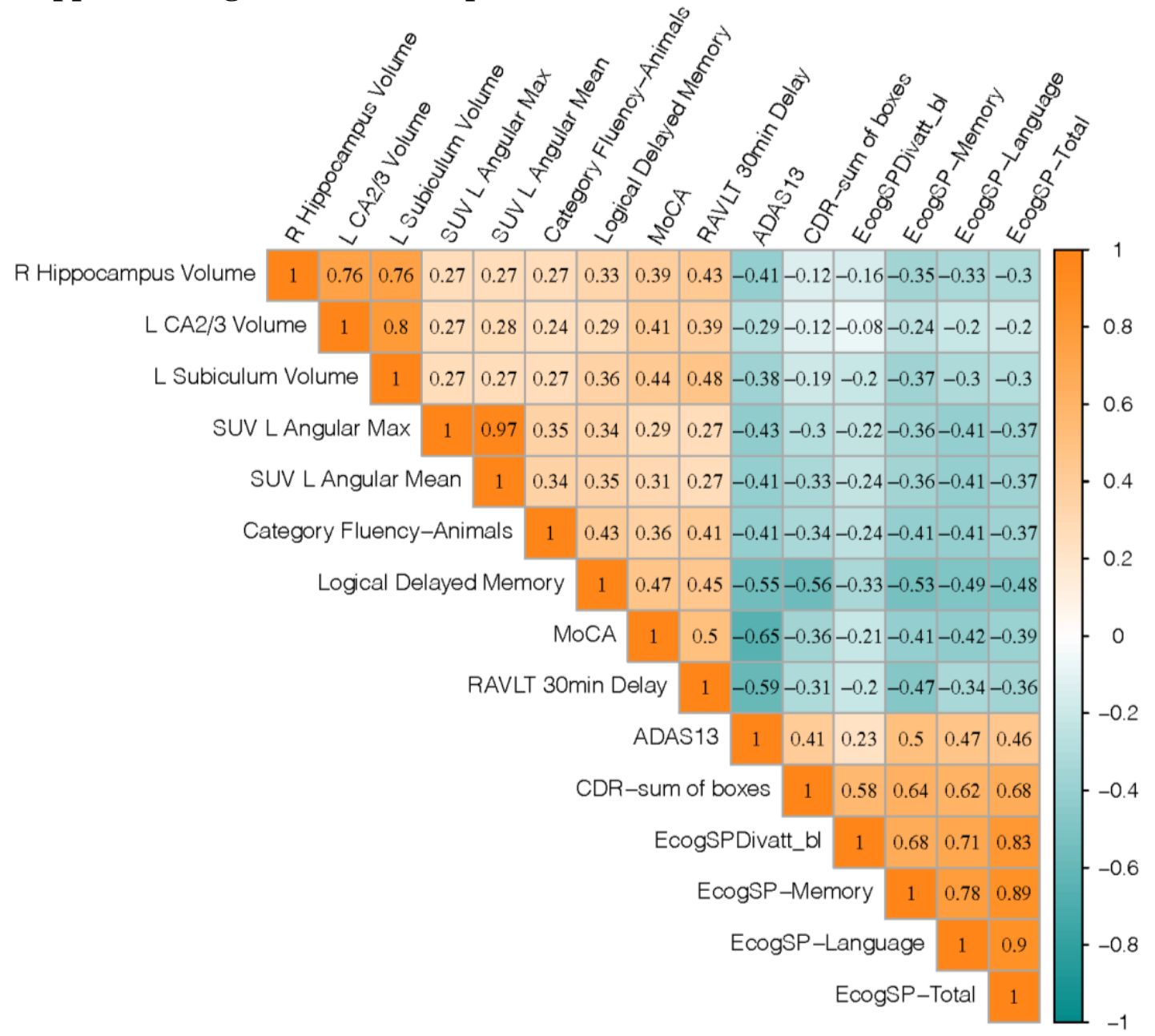

Abbreviations: ADAS13=Alzheimer's Disease Assessment Scale; $B L=$ bilateral; $C D R=$ Clinical Dementia Rating; Ecog=Everyday cognition-Study Partner; L=left; MoCA=Montreal Cognition Assessment; R=right; RAVLT=Ray Auditory Verbal Learning Test 


\section{Supplement Fig 2. Plot of c parameter with repeated k-fold cross-validation}
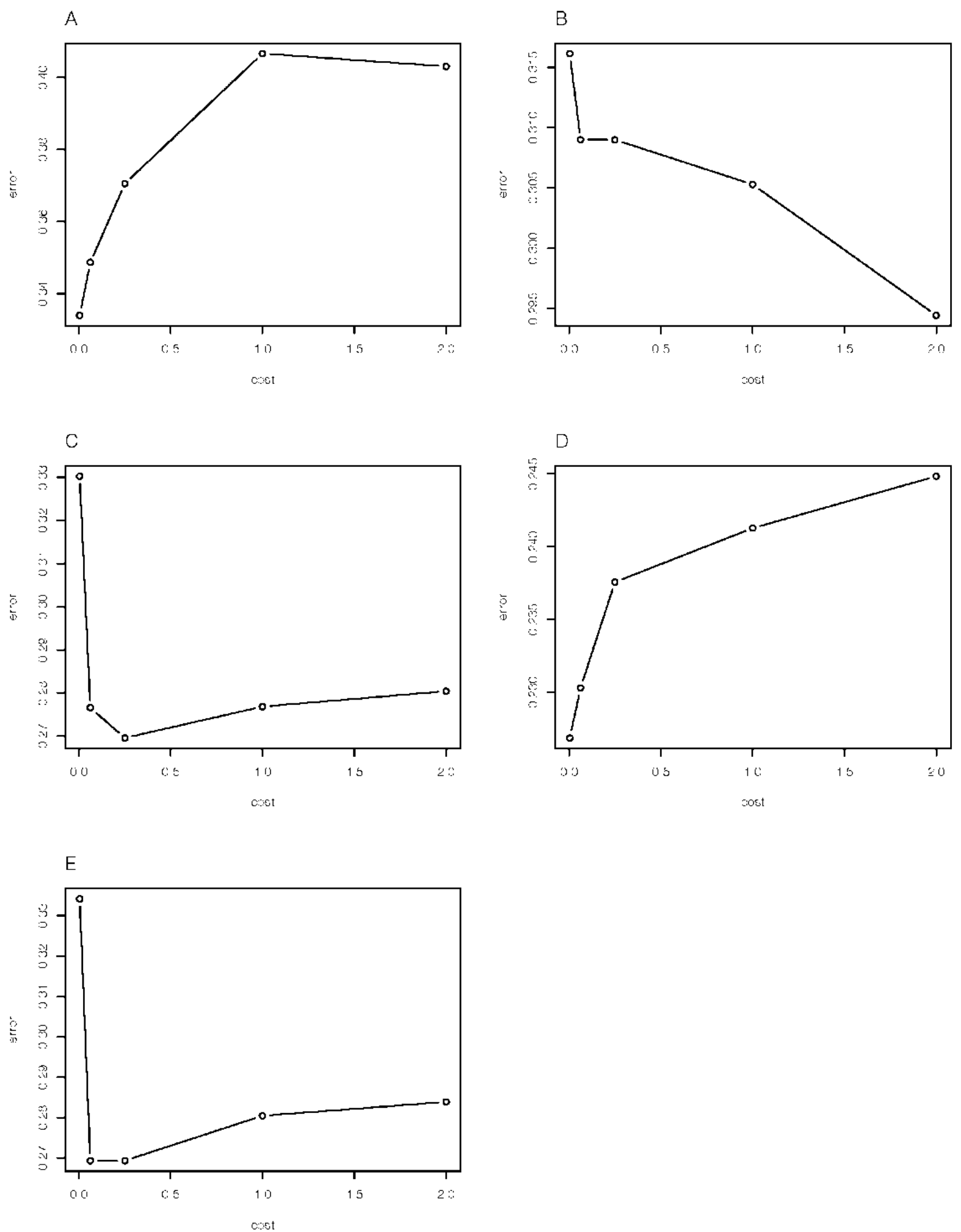

Tuning of c parameter occurred in the training sample prior to model evaluation in the testing sample. $\mathrm{C}$ parameter was tuned with repeated $\mathrm{k}$-fold cross-validation in which $\mathrm{k}=10$ with 50 repeats. Values of $\mathrm{c}$ tested were $2^{-8}, 2^{-4}, 2^{-2}, 1,2,16$.

A Neurocognitive measures

B FDG-PET measures

C Genetics/fluid-based biomarkers

D MRI measures

E Demographic information

Abbreviations: FDG=fluorodeoxyglucose 
Albright J. Forecasting the progression of Alzheimer's disease using neural networks and a novel preprocessing algorithm. Alzheimers Dement Transl Res Clin Interv 2019; 5: 483-91

Apostolova LG, Hwang KS, Avila D, Elashoff D, Kohannim O, Teng E, et al. Brain amyloidosis ascertainment from cognitive, imaging, and peripheral blood protein measures. Neurology 2015; 84: 729-37.

Ba M, Li X, Ng KP, Pascoal TA, Mathotaarachchi S, Rosa-Neto P et al. The prevalence and biomarkers' characteristic of rapidly progressive Alzheimer's disease from the Alzheimer's Disease Neuroimaging Initiative database. Alzheimers Dement 2017; 3: 107-13.

Berg L. Clinical Dementia Rating (CDR). Psychopharmacol Bull 1988; 24: 637-9.

Bucholc M, Ding X, Wang H, Glass DH, Wang H, Prasad G, et al. A practical computerized decision support system for predicting the severity of Alzheimer's disease of an individual. Expert Syst Appl 2019; 130: 157-71.

Buckner RL, Head D, Parker J, Fotenos AF, Marcus D, Morris JC, et al. A unified approach for morphometric and functional data analysis in young, old, and demented adults using automated atlas-based head size normalization: reliability and validation against manual measurement of total intracranial volume. NeuroImage 2004; 23: 724-38.

Cabeza R, Ciaramelli E, Moscovitch M. Cognitive contributions of the ventral parietal cortex: an integrative theoretical account. Trends Cogn Sci 2012; 16: 338-52.

Carter JV, Pan J, Rai SN, Galandiuk S, ROC-ing along: Evaluation and interpretation of receiver operating characteristic curve. Surgery 2016; 6: 1638-45.

Christensen K, Doblhammer G, Rau R, Vaupel JW. Ageing populations: the challenges ahead. Lancet 2009; 374: 1196-208.

Cui Y, Liu B, Luo S, Zhen X, Fan M, Liu T, et al. Identification of conversion from mild cognitive impairment to Alzheimer's disease using multivariate predictors. PloS One 2011; 6: e21896.

Dale A, Fischl B, Sereno MI. Cortical surface-based analysis: I. Segmentation and surface reconstruction. NeuroImage 1999; 9: 179-94.

Dale AM, Sereno MI. Improved localizadon of cortical activity by combining EEG and MEG with MRI cortical surface reconstruction: a linear approach. J Cogn Neurosci 1993; 5: 16276.

Desikan RS, Fan CC, Wang Y, Schork AJ, Cabral HJ, Cupples LA, et al. Genetic assessment of age-associated Alzheimer disease risk: Development and validation of a polygenic hazard score. PLoS Med 2017; 14: e1002258.

Dowling NM, Bolt DM, Deng S. An approach for estimating item sensitivity to withinperson change over time: An illustration using the Alzheimer's Disease Assessment ScaleCognitive subscale (ADAS-Cog). Psychol Assess 2016; 28: 1576-85. 
Emch M, von Bastian CC, Koch K. Neural Correlates of Verbal Working Memory: An fMRI Meta-Analysis. Front Hum Neurosci 2019; 13

Farias ST, Cahn-Weiner DA, Harvey DJ, Reed BR, Mungas D, Kramer JH, et al. Longitudinal Changes in Memory and Executive Functioning are Associated with Longitudinal Change in Instrumental Activities of Daily Living in older adults. Clin Neuropsychol 2009; 23: 446-61.

Farias ST, Mungas D, Reed BR, Cahn-Weiner D, Jagust W, Baynes K, et al. The measurement of everyday cognition (ECog): scale development and psychometric properties. Neuropsychology 2008; 22: 531-44.

Fischl B, Dale AM. Measuring the thickness of the human cerebral cortex from magnetic resonance images. Proc Natl Acad Sci U S A 2000; 97: 11050-5.

Fischl B, van der Kouwe A, Destrieux C, Halgren E, Ségonne F, Salat DH, et al. Automatically parcellating the human cerebral cortex. Cereb Cortex 2004; 14: 11-22.

Fischl B, Liu A, Dale AM. Automated manifold surgery: constructing geometrically accurate and topologically correct models of the human cerebral cortex. IEEE Med Imaging 2001; 20: $70-80$.

Fischl B, Salat DH, Busa E, Albert M, Dieterich M, Haselgrove C, et al. Whole brain segmentation: automated labeling of neuroanatomical structures in the human brain. Neuron 2002; 33: 341-55.

Fischl B, Salat DH, van der Kouwe AJW, Makris N, Ségonne F, Quinn BT, et al. Sequenceindependent segmentation of magnetic resonance images. NeuroImage 2004; 23: S69-84.

Fischl B, Sereno MI, Dale A. Cortical surface-based analysis: II: inflation, flattening, and a surface-based coordinate system. NeuroImage 1999; 9: 195-207.

Fischl B, Sereno MI, Tootell RBH, Dale AM. High-resolution intersubject averaging and a coordinate system for the cortical surface. Hum Brain Mapp 1999; 8: 272-284.

Folstein MF, Folstein SE, McHugh PR. 'Mini-mental state'. A practical method for grading the cognitive state of patients for the clinician. J Psychiatr Res 1975; 12: 189-98.

Goodglass H, Kaplan E. The assessment of aphasia and related disorders. Second. Lea \& Febiger; 1982

Gray KR, Aljabar P, Heckemann RA, Hammers A, Rueckert D, Alzheimer's Disease Neuroimaging Initiative. Random forest-based similarity measures for multi-modal classification of Alzheimer's disease. NeuroImage 2013; 65: 167-75.

Griffith HR, Stewart CC, Stoeckel LE, Okonkwo OC, den Hollander JA, Martin RC, et al. MRI Volume of the Angular Gyri Predicts Financial Skill Deficits in Patients with Amnestic Mild Cognitive Impairment. J Am Geriatr Soc 2010; 58: 265-74.

Gunter JL, Bernstein MA, Borowski BJ, Ward CP, Britson PJ, Felmlee JP. Measurement of MRI scanner performance with the ADNI phantom. Med Phys 2009; 36: 2193-205. 
Han X, Jovicich J, Salat D, van der Kouwe A, Quinn B, Czanner S, et al. Reliability of MRIderived measurements of human cerebral cortical thickness: the effects of field strength, scanner upgrade and manufacturer. NeuroImage 2006; 32: 180-94.

Hayes SM, Buchler N, Stokes J, Kragel J, Cabeza R. Neural Correlates of Confidence during Item Recognition and Source Memory Retrieval: Evidence for Both Dual-process and Strength Memory Theories. J Cogn Neurosci 2011; 23: 3959-71.

Houdé O, Rossi S, Lubin A, Joliot M. Mapping numerical processing, reading, and executive functions in the developing brain: an fMRI meta-analysis of 52 studies including 842 children. Dev Sci 2010; 13: 876-85.

Hutchinson JB, Uncapher MR, Wagner AD. Posterior parietal cortex and episodic retrieval: Convergent and divergent effects of attention and memory. Learn Mem 2009; 16: 343-56.

Jack CR Jr, Bernstein MA, Borowski BJ, Gunter JL, Fox NC, Thompson PM, et al. Update on the magnetic resonance imaging core of the Alzheimer's disease neuroimaging initiative. Alzheimers Dement 2010; 6: 212-20.

Jovicich J, Czanner S, Greve D, Haley E, van der Kouwe A, Gollub R, et al. Reliability in multi-site structural MRI studies: effects of gradient non-linearity correction on phantom and human data. NeuroImage 2006; 30: 436-43.

Kalpouzos G, Eriksson J, Sjölie D, Molin J, Nyberg L. Neurocognitive systems related to real-world prospective memory. PloS One 2010; 5: e13304.

Kaplan E, Goodglass H, Weintraub S, Goodglass H. Boston naming test. Philadelphia: Lea \& Febiger; 1983

Kaur N, Belchior P, Gelinas I, Bier N. Critical appraisal of questionnaires to assess functional impairment in individuals with mild cognitive impairment. Int Psychogeriatr 2016; 28: 142539.

Kuhn M. caret: Classification and Regression Training [Internet]. 2020Available from: https://CRAN.R-project.org/package=caret

Landau SM, Harvey D, Madison CM, Koeppe RA, Reiman EM, Foster NL, et al. Associations between cognitive, functional, and FDG-PET measures of decline in AD and MCI. Neurobiol Aging 2011; 32: 1207-18.

Lewis CM, Vassos E. Prospects for using risk scores in polygenic medicine. Genome Med 2017; 9

Ling CX, Huang J, Zhang H. AUC: a statistically consistent and more discriminating measure than accuracy. In: Ijcai. 2003. p. 519-24

López SR, Montero P, Carmenate M, Avendano M. Functional decline over 2 years in older Spanish adults: Evidence from the Survey of Health, Ageing and Retirement in Europe. Geriatr Gerontol Int 2014; 14: 403-12. 
Malek-Ahmadi M, Chen K, Davis K, Belden CM, Powell J, Jacobson SA, et al. Sensitivity to change and prediction of global change for the Alzheimer's Questionnaire. Alzheimers Res Ther 2015; 7: 1 .

Mar RA. The neural bases of social cognition and story comprehension. Annu Rev Psychol 2011; 62: 103-34.

McKhann GM, Knopman DS, Chertkow H, Hyman BT, Jack CR, Kawas CH, et al. The diagnosis of dementia due to Alzheimer's disease: Recommendations from the National Institute on Aging-Alzheimer's Association workgroups on diagnostic guidelines for Alzheimer's disease. Alzheimers Dement 2011; 7: 263-9.

Mihaescu AS, Masellis M, Graff-Guerrero A, Kim J, Criaud M, Cho SS, et al. Brain degeneration in Parkinson's disease patients with cognitive decline: a coordinate-based metaanalysis. Brain Imaging Behav 2019; 13: 1021-34.

Mohs RC, Knopman D, Petersen RC, Ferris SH, Ernesto C, Grundman M, Sano M, et al. Development of cognitive instruments for use in clinical trials of antidementia drugs: Additions to the Alzheimer's Disease Assessment Scale that broaden its scope. Alzheimer Dis Assoc Disord 1997.

Nasreddine ZS, Phillips NA, Bédirian V, Charbonneau S, Whitehead V, Collin I, et al. The Montreal Cognitive Assessment, MoCA: a brief screening tool for mild cognitive impairment. J Am Geriatr Soc 2005; 53: 695-9.

Nelson HE, O'Connell A. Dementia: the estimation of premorbid intelligence levels using the New Adult Reading Test. Cortex J Devoted Study Nerv Syst Behav 1978; 14: 234-44.

Nosheny RL, Jin C, Neuhaus J, Insel PS, Mackin RS, Weiner MW. Study partner-reported decline identifies cognitive decline and dementia risk. Ann Clin Transl Neurol 2019; 6: 2448-59.

Ohio Supercomputer Center. Ohio Supercomputer Center. Columbus OH: Ohio Supercomputer Center. 1987

Okonkwo OC, Alosco ML, Griffith HR, Mielke MM, Shaw LM, Trojanowski JQ, et al. Cerebrospinal fluid abnormalities and rate of decline in everyday function across the dementia spectrum: normal aging, mild cognitive impairment, and Alzheimer disease. Arch Neurol 2010; 67: 688-96.

Okonkwo OC, Alosco ML, Jerskey BA, Sweet LH, Ott BR, Tremont G, et al. Cerebral atrophy, apolipoprotein $\mathrm{E}$ varepsilon4, and rate of decline in everyday function among patients with amnestic mild cognitive impairment. Alzheimers Dement J Alzheimers Assoc 2010; 6: 404-11.

Peduzzi P, Concato J, Feinstein AR, Holford TR. Importance of events per independent variable in proportional hazards regression analysis II. Accuracy and precision of regression estimates. J Clin Epidemiol 1995; 48: 1503-10. 
Peduzzi P, Concato J, Kemper E, Holford TR, Feinstein AR. A simulation study of the number of events per variable in logistic regression analysis. J Clin Epidemiol 1996; 49: 1373-79.

Pérès K, Helmer C, Amieva H, Orgogozo J-M, Rouch I, Dartigues J-F, et al. Natural History of Decline in Instrumental Activities of Daily Living Performance over the 10 Years Preceding the Clinical Diagnosis of Dementia: A Prospective Population-Based Study. J Am Geriatr Soc 2008; 56: 37-44.

Pfeffer RI, Kurosaki TT, Harrah CH, Chance JM, Filos S. Measurement of Functional Activities in Older Adults in the Community. J Gerontol 1982; 37: 323-9.

Qiu C, Kivipelto M, von Strauss E. Epidemiology of Alzheimer's disease: occurrence, determinants, and strategies toward intervention. Dialogues Clin Neurosci 2009; 11: 111-28.

Rabaglia CD, Salthouse TA. Natural and constrained language production as a function of age and cognitive abilities. Lang Cogn Process 2011; 26

RC Team. R: A language and environment for statistical computing. 2013

Reitan RM. Validity of the Trail Making Test as an indicator of organic brain damage. Percept Mot Skills 1958; 8: 271-6.

Reuter M, Rosas HD, Fischl B. Highly accurate inverse consistent registration: a robust approach. NeuroImage 2010; 53: 1181-1196.

Reuter M, Schmansky NJ, Rosas HD, Fischl B. Within-subject template estimation for unbiased longitudinal image analysis. NeuroImage 2012; 61: 1402-1418.

Rey A. L'examen psychologique dans les cas d'encéphalopathie traumatique.(Les problems.). Arch Psychol 1941

Ritter K, Schumacher J, Weygandt M, Buchert R, Allefeld C, Haynes J-D. Multimodal prediction of conversion to Alzheimer's disease based on incomplete biomarkers. Alzheimers Dement Diagn Assess Dis Monit 2015; 1: 206-15.

Rosen WG. Verbal fluency in aging and dementia. J Clin Neuropsychol 1980; 2: 135-46.

Rosen WG, Mohs RC, Davis KL. A new rating scale for Alzheimer's disease. Am J Psychiatry 1984; 141: 1356-64.

Royall DR, Palmer R, Chiodo LK, Polk MJ. Declining executive control in normal aging predicts change in functional status: the Freedom House Study. J Am Geriatr Soc 2004; 52 : 346-52.

Rudner M, Signoret C. The Role of Working Memory and Executive Function in Communication under Adverse Conditions. Frontiers Media SA; 2016

Salthouse TA. Selective review of cognitive aging. J Int Neuropsychol Soc JINS 2010; 16: 754-60. 
Sano M, Raman R, Emond J, Thomas RG, Petersen R, Schneider LS, et al. Adding delayed recall to the Alzheimer's Disease Assessment Scale is useful in studies of mild cognitive impairment but not Alzheimer's disease. Alzheimer Dis Assoc Disord 2011; 25: 122-27.

Saykin AJ, Wishart HA, Rabin LA, Santulli RB, Flashman LA, West JD, et al. Older adults with cognitive complaints show brain atrophy similar to that of amnestic MCI. Neurology 2006; 67: 834-42.

Schumann G, Benegal V, Yu C, Tao S, Jernigan T, Heinz A, et al. Precision medicine and global mental health. Lancet Glob Health 2019; 7: e32.

Seeman TE, Merkin SS, Crimmins EM, Karlamangla AS. Disability Trends Among Older Americans: National Health and Nutrition Examination Surveys, 1988-1994 and 1999-2004. Am J Public Health 2010; 100: 100-7.

Seghier ML. The Angular Gyrus. The Neuroscientist 2013; 19: 43-61.

Segonne F, Dale AM, Busa E, Glessner M, Salat D, Hahn HK, et al. A hybrid approach to the skull stripping problem in MRI. NeuroImage 2004; 22: 1060-75.

Segonne F, Pacheco J, Fischl B. Geometrically accurate topology-correction of cortical surfaces using nonseparating loops. IEEE Trans Med Imaging 2007; 26: 518-29.

Sled JG, Zijdenbos AP, Evans AC. A nonparametric method for automatic correction of intensity nonuniformity in MRI data. IEEE Trans Med Imaging 1998; 17: 87-97.

Tabert MH, Albert SM, Borukhova-Milov L, Camacho Y, Pelton G, Liu X, et al. Functional deficits in patients with mild cognitive impairment. Neurology 2002

Teng E, Tassniyom K, Lu PH. Reduced Quality-of-Life Ratings in Mild Cognitive Impairment: Analyses of Subject and Informant Responses. Am J Geriatr Psychiatry 2012; 20: $1016-25$.

Thomas KR, Edmonds EC, Delano-Wood L, Bondi MW. Longitudinal Trajectories of Informant-Reported Daily Functioning in Empirically Defined Subtypes of Mild Cognitive Impairment. J Int Neuropsychol Soc JINS 2017; 23: 521-7.

Tomasi D, Volkow ND. Association between Functional Connectivity Hubs and Brain Networks. Cereb Cortex N Y NY 2011; 21: 2003-13.

Trojanowski JQ, Vandeerstichele H, Korecka M, Clark CM, Aisen PS, Petersen RC, et al. Update on the biomarker core of the Alzheimer's Disease Neuroimaging Initiative subjects. Alzheimers Dement 2010; 6: 230-8.

Vaughan L, Leng X, La Monte MJ, Tindle HA, Cochrane BB, Shumaker SA. Functional Independence in Late-Life: Maintaining Physical Functioning in Older Adulthood Predicts Daily Life Function after Age 80. J Gerontol A Biol Sci Med Sci 2016; 71: S79-86.

Vigneau M, Beaucousin V, Hervé PY, Duffau H, Crivello F, Houdé O, et al. Meta-analyzing left hemisphere language areas: phonology, semantics, and sentence processing. NeuroImage 2006; 30: 1414-32. 
Predicting functional decline

Wechsler D. Wechsler memory scale-revised. Psychol Corp 1987

Willet JB, Singer JD. Another Cautionary Note About $\mathrm{R}^{2}$ : Its Use in Weighted Least-Squares Regression Analysis. The American Statistician 1988; 42: 236-38.

Zhou B, Nakatani E, Teramukai S, Nagai Y, Fukushima M. Risk classification in mild cognitive impairment patients for developing Alzheimer's disease. J Alzheimers Dis 2012; 30: $367-75$. 